\title{
Nivel de conocimientos sobre salud oral e índice de caries en adolescentes de una escuela de Pirayú, Paraguay
}

\author{
Knowledge level of oral health and rates of caries in adolescents \\ from a school in Pirayú, Paraguay
}

\author{
Milner Morel-Barrios ${ }^{1}$, Clarisse Díaz-Reissner ${ }^{1}$, María Elida Quintana-Molinas ${ }^{1}$
}

\section{RESUMEN}

Introducción: La caries dental continúa siendo un problema de salud pública en países subdesarrollados, siendo una de las causas más importantes de problemas bucodentales en la adolescencia. Tener conocimientos sobre medidas de cuidado de salud bucal se asoció con un mejor estado de salud bucal. Objetivo: Relacionar el nivel de conocimientos sobre salud oral con el índice de caries dental en adolescentes de 12 a 15 años, matriculados en la Escuela Básica №80 “General José Eduvigis Díaz" de Pirayú (Paraguay), en el año 2016. Material y Métodos: El diseño fue transversal. Se realizó una evaluación clínica utilizando el Índice CPO-D y además 12 preguntas sobre conocimiento de salud oral. La escala de puntajes fue de 0 a 28, donde valores elevados indicaron mayor conocimiento y puntajes $60 \%$ se consideraron con conocimiento suficiente. Se aplicaron las pruebas $t$ de Student y U de Mann-Whitney, con un nivel de confianza del 95\%. Resultados: Participaron 70 adolescentes, el $62,9 \%$ fueron varones y el 37,1\% fueron mujeres. El índice CPO-D fue de 2,24 y el índice de caries de 1,17. Presentaron al menos un diente perdido el $42,8 \%$ de los adolescentes. El $93,98 \%$ de pérdidas dentarias correspondió a primeros molares permanentes. El puntaje promedio de conocimiento fue 17,31 (DE=4,48). E154,30\% tuvieron nivel de conocimientos suficiente. Conclusión: El nivel de conocimientos no presentó relación con el índice de caries ni con el índice CPO-D, el nivel de severidad de caries fue de bajo riesgo, pero un poco menos de la mitad perdió al menos un diente permanente. El nivel de conocimientos sobre salud oral fue suficiente en un poco más de la mitad. Se deberían reforzar los programas de prevención primaria y secundaria, a fin de evitar pérdidas dentarias a temprana edad.

\begin{abstract}
Introduction: Dental caries continues to be a public health problem in underdeveloped countries and is one of the most important causes of oral dental problems in adolescence. Having knowledge regarding oral health care has been associated with improved oral health status. Objective: To correlate the level of knowledge regarding oral health with the rate of dental caries in adolescents aged 12 to 15 years, enrolled in Basic School No.80 "General José Eduvigis Díaz" in Pirayú (Paraguay), in 2016. Materials and Methods: This was a cross-sectional study.. A clinical evaluation was performed using the DMF-T Index plus 12 questions about oral health knowledge. The scale of scores was from 0 to 28 , where high values indicated greater knowledge and scores of $60 \%$ were considered sufficient knowledge. Student's t test and Mann-Whitney U test were applied, with a confidence level of $95 \%$. Results: 70 adolescents participated, $62.9 \%$ were male and $37.1 \%$ were female. The DMF-T index was 2.24 and the caries index was 1.17. $42.8 \%$ of adolescents presented with at least one lost tooth. $93.98 \%$ of dental losses corresponded to permanent first molars. The average knowledge score was $17.31(\mathrm{SD}=4.48) .54 .3 \%$ had a sufficient knowledge level. Conclusion: The level of knowledge was not related to the caries index or the DMF$\mathrm{T}$ index, the level of caries severity was low risk, but slightly less than half had lost at least one permanent tooth. The level of knowledge about oral health sufficient in little more than half of the subjects. Primary and secondary prevention programs should be reinforced in order to avoid dental losses at such an early age.
\end{abstract}

Keywords: Dental caries, knowledge, adolescents, oral Health, Paraguay

Palabras clave: Caries dental, Conocimiento, Adolescentes, Salud Bucal, Paraguay

\footnotetext{
${ }^{1}$ Universidad Nacional de Asunción, Facultad de Odontología. Asunción, Paraguay.

Correspondencia: Dra. Clarisse Díaz Reissner; Correo: cdiazr@odo.una.py

Conflicto de interés: Los autores declaran no tener conflicto de intereses.

Recibido: 25/10/2018; Aceptado: 27/11/2018

DOI: https://doi.org/10.31698/ped.45032018004
} 


\section{INTRODUCCIÓN}

Según la Organización Mundial de la Salud (OMS) la adolescencia "es el periodo de crecimiento y desarrollo humano que se produce después de la niñez y antes de la edad adulta, de 10 a 19 años, y es una de las etapas de transición más importantes en la vida del ser humano ${ }^{(1)}$. Los adolescentes sufren cambios físicos y emocionales; dada esta situación es indispensable poner atención sobre distintos aspectos de la salud oral y general; por consiguiente esta etapa es la ideal para la implementación de buenos hábitos, de manera a posibilitar una óptima salud oral ${ }^{(2)}$.

Los adolescentes son propensos a presentar problemas de salud oral ${ }^{(3)}$, siendo la caries dentales una de las causas más importantes de dichos problemas. La mala higiene oral contribuye la aparición de la caries dental y se encuentra asociada a alimentación inadecuada, pocas horas de sueño y falta confianza en sí mismo ${ }^{(4)}$.Como resultado, la presencia de patologías orales pueden impactar negativamente en la calidad de vida de los adolescentes $^{(5)}$. De ahí que la salud oral se debe considerar como parte del desarrollo integral, ya que se consideran una población de riesgo, por dicho motivo se sugiere que cuenten con acompañamiento continúo de la familia, la sociedad y el estado( ${ }^{(6)}$.

En el reporte de "Salud bucal de las Américas" se determinó que existen disparidades en el nivel de salud oral, lo que lleva a pensar que esta epidemia silenciosa afecta a grupos específicos de la población $^{(7)}$. Con respecto a la caries dental a los 12 años, Paraguay en el año 2008 presentaba un índice CPO-D de riesgo moderado con un valor de $2,79^{(8)} \mathrm{y}$ en el año 2017 el riesgo fue bajo con un valor de $2,07^{(9)}$.

Al mismo tiempo, tener mayores conocimientos sobre salud oral redundará en cambios de actitudes positivas y motivación para el desarrollo de conductas saludables aumentando la predisposición del individuo para realizar tareas de prevención oral ${ }^{(10,11)}$. Así también, tener conocimientos acerca de salud oral se asoció con mejores prácticas de medidas de higiene oral ${ }^{(12)}$, pues esto ayudaría a prevenir enfermedades orales ${ }^{(13)}$. Por tanto, el cambio e implementación de hábitos saludables debe ser parte del contexto de vida individual y colectiva ${ }^{(14)}$.

En este estudio se planteó como objetivo relacionar el nivel de conocimiento sobre salud oral con el índice de caries dental en adolescentes de 12 a 15 años, matriculados en la Escuela Básica № 80 “General José Eduvigis Díaz" de Pirayú (Paraguay), en el año 2016. Si bien, los adolescentes sufren cambios trascendentales en el proceso de ser adultos, es en esta población donde se debería reforzar los mecanismos de la educación para la salud y la adquisición de buenas prácticas. Implementar un programa sostenido de hábitos de higiene oral, alimentación saludable e identificación de factores de riesgo para caries y enfermedad periodontal, resultará en una salud integral del individuo.

\section{MATERIALES Y MÉTODOS}

Se realizó un estudio transversal, con 70 adolescentes de 12 a 15 años de la Escuela Básica №80 "General José Eduvigis Díaz" de la ciudad de Pirayú del Departamento de Paraguarí-Paraguay, matriculados en el año 2016 del 7mo al 9no grado, de los turnos mañana y tarde. Las variables consideradas fueron: conocimientos sobre salud bucal, índice CPO-D, índice de caries dental, edad en años y sexo.

Se entregó un cuestionario con 12 preguntas sobre conocimientos de salud bucal relacionado a caries dental, gingivitis, higiene bucodental, cariogenicidad de los alimentos, acción del flúor en la cavidad bucal y permanencia de los dientes en boca. Se utilizó el cuestionario de Dho ${ }^{(15)}$, que fue aplicado en adultos correntinos en Argentina, que analizó los conocimientos de salud bucal en relación con el nivel socioeconómico. El puntaje de la escala fue de 0 a 28 puntos, cuanto más elevado el puntaje indicó mayor conocimiento. Se consideraron con conocimiento suficiente puntajes $60 \%$ de respuestas correctas, lo que equivale a 17 puntos. Se realizó la inspección bucal siguiendo los criterios de la OMS, estandarizados para este tipo de evaluaciones orales ${ }^{(7)}$.

Se realizaron charlas sobre prevención de la salud como parte del programa del Ministerio de Salud y 
Bienestar Social. Se contó con la ayuda del odontólogo encargado de dicha escuela, previamente entrenado. Se solicitó autorización del director de la escuela y a los docentes. Los padres consintieron la participación y formaron parte aquellos adolescentes que asintieron formar parte del estudio.

Los resultados se presentaron en tablas, se utilizaron medidas de tendencia central y dispersión para variables cuantitativas; frecuencia y porcentaje para variables cuantitativas. Se aplicaron las pruebas t de Student previa comprobación de normalidad con la prueba de Kolmogorov-Smirnov y homogeneidad de varianza con el test de Levene para contrastar la puntuación promedio del conocimiento por sexo, la prueba U de Mann-Whitney para contrastar índice CPO-D por sexo, índice CPO-D por nivel de conocimiento e índice de caries por nivel de conocimiento. Todos con un nivel de confianza del 95\%. Se utilizaron los programas Microsoft Excel e InfoStat/L.

\section{RESULTADOS}

Participaron del estudio 70 adolescentes de 12 a 15 años matriculados en la escuela pública de la ciudad de Pirayú. El 18,6\% tenía 12 años, el 34,3\% tenía 13 años, el 31,4\% tenía 14 años y el 15,7\% tenía 15 años. el $62,9 \%$ fueron varones y el $37,1 \%$ fueron mujeres. El índice CPO-D fue de 2,24 indicativo de bajo riesgo y el índice de caries de 1,17. Presentaron al menos un diente perdido el $42,8 \%$ de los adolescentes (Gráfico 1).

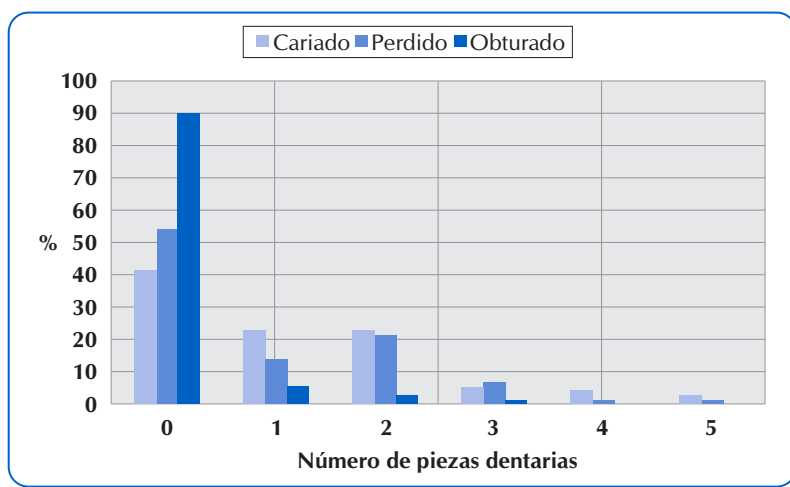

Gráfico 1. Componentes del Índice CPO-D por número de piezas dentarias en adolescentes de Pirayú, Paraguay. Año 2016.

El puntaje promedio fue de $17,31(\mathrm{DE}=4,48)$ en el cuestionario de nivel de conocimientos sobre salud oral. El 45,7\% obtuvieron conocimientos insuficientes (Gráfico 2).

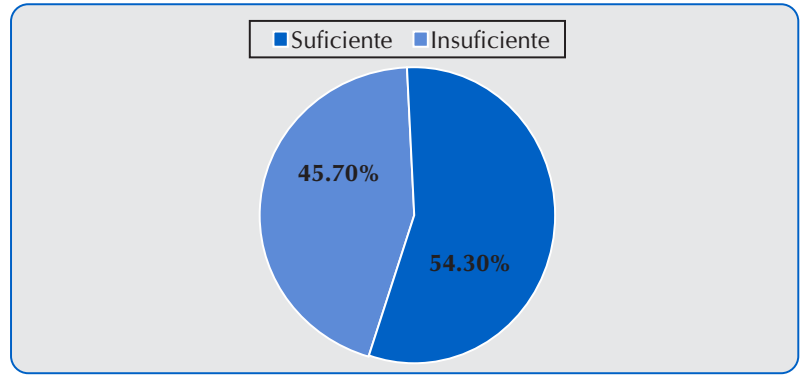

Gráfico 2. Distribución del nivel de conocimientos sobre salud oral en adolescentes de Pirayú, Paraguay. Año 2016.

No se encontraron diferencias estadísticamente significativas al comparar la distribución del puntaje promedio de conocimientos e índice CPO-D por sexo ni al comparar por edad (Tabla 1).

Tabla 1. Distribución del nivel de conocimientos sobre salud oral e Índice CPOD-D por edad y sexo en adolescentes de Pirayú, Paraguay. Año 2016.

\begin{tabular}{lccccc}
\hline Variables & \multicolumn{2}{c}{ Conocimientos sobre salud oral } & \multicolumn{2}{c}{ Indice CPO-D } \\
\cline { 2 - 6 } & Media & DE & p-valor & Media & p-valor \\
\hline Edad (años) & & & & & \\
12 a $13(\mathrm{n}=37)$ & 17,27 & 4,22 & 0,937 & 2,14 & 0,411 \\
14 a $15(\mathrm{n}=33)$ & 17,36 & 5,53 & & 2,36 & \\
Sexo & & & & & \\
$\quad$ Masculino $(\mathrm{n}=44)$ & 17,7 & 5,29 & 0,385 & 2,24 & 0,814 \\
$\quad$ Femenino $(\mathrm{n}=26)$ & 16,65 & 4 & & 2,33 & \\
TOTAL & $\mathbf{1 7 , 3 1}$ & $\mathbf{4 , 4 8}$ & & $\mathbf{2 , 2 4}$ & \\
\hline
\end{tabular}

Presentaron al menos un diente perdido el $42,8 \%$ de los adolescentes. El 93,98\% de pérdidas dentarias correspondió a primeros molares permanentes. En cuanto al tipo de piezas dentarias, los molares fueron los dientes más perdidos (Gráfico 3).

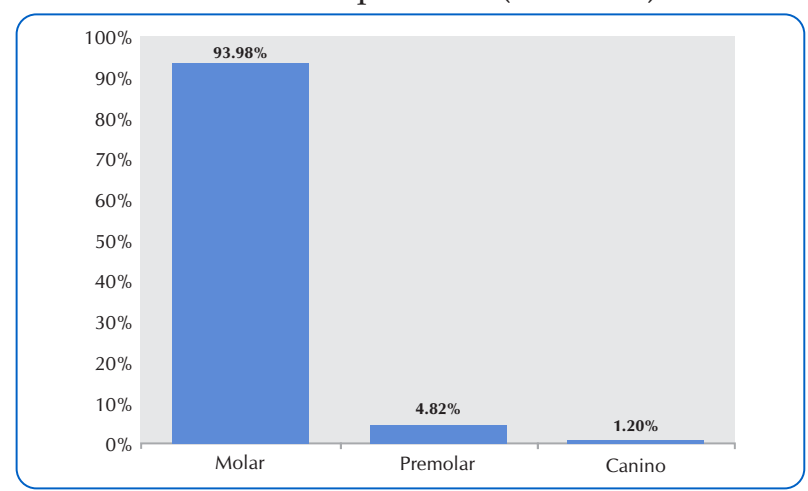

Gráfico 3. Distribución del tipo piezas dentarias perdidas en adolescentes de Pirayú, Paraguay. Año 2016. 
El índice CPO-D por nivel de conocimientos sobre salud oral no resultó estadísticamente significativo $(\mathrm{p}=0,723)$, así como tampoco el índice de caries por nivel de conocimientos $(\mathrm{p}=0,590)$.

\section{DISCUSIÓN}

Se planteó como objetivo relacionar el nivel de conocimientos sobre salud oral con el índice de caries dental en adolescentes de 12 a 15 años, matriculados en una escuela de Pirayú, Paraguay en el año 2016, pero no se encontró relación entre el índice de caries y el nivel de conocimientos sobre salud oral.

En base a las respuestas de los adolescentes, del estudio el 45,70\% tiene conocimientos insuficientes sobre los causales de caries e incluso como mejorar esta situación, mientras que un estudio similar realizado en adolescentes paquistaníes presentaron resultados semejantes ${ }^{(16)}$, por lo cual se debería insistir en la responsabilidad para con su propia salud oral, pudiéndose indicar la necesidad de insistir en programas preventivos apoyados por los docentes y las familias. Con respecto a los factores que favorecen la aparición de caries dental, el 100\% marcó las opciones correspondientes al consumo de azúcar y bacterias, y un $71,4 \%$ a la mala higiene; mientras que en el Valle del Cauca, Colombia los adolescentes atribuyen a la mala higiene un $65 \%$, a las bacterias un $18 \%$ y al consumo de azúcar un $7 \%{ }^{(17)}$. El 50\% de los adolescentes paraguayos utiliza hilo dental en coincidencia con poblaciones similares de brasileros $(51 \%)^{(18)}$ y colombianos $(55 \%)^{(19)}$.

El índice CPO-D obtenido en este estudio $(2,24)$ fue ligeramente superior al obtenido a nivel nacional en el año 2017 en adolescentes de 12 años pero ambos presentan riesgo bajo $^{(9)}$. Pero fue superior al índice CPO-D en niños mexicanos de 12 años $(1,4 \pm 1,7)^{(19)}$, muy superior al índice de niños de 6 a 12 años de la ciudad de Sátão, Portugal $(0,93 \pm 0,63)^{(20)}$, similar al índice niños de 12 años que acudieron a escuelas públicas y privadas dede Santa Cruz do Sul, Rio Grande do Sul, Brasil $(2,5)^{(21)}$, ligeramente inferior a adolescentes de 18 años lituanos $(2,93 \pm 2,81)^{(2)}$. Asimismo, en este estudio el índice CPO-D se encuentra en un nivel de riesgo de caries bajo, mientras que Sanabria-Castellanos et al. reportan nivel intermedio ${ }^{(8)}$, en el mismo nivel que Alaska, Perú, Venezuela, Chile, Ecuador, España, Italia, Rusia y Uruguay. El índice de caries de este estudio $(1,17)$ fue ligeramente inferior al 1,40 reportado en niños y adolescentes en orfanatos de la ciudad de Jodhpur en Rajasthan, India ${ }^{(23)}$.

Es importante destacar que un elevado porcentaje $(93,98 \%)$ de perdidas dentarias corresponde a los primeros molares permanentes, se debe considerar su importancia debido a que constituyen las piezas dentarias muy importantes para la masticación y su presencia resulta fundamental para el correcto desarrollo de la oclusión ${ }^{(24)}$. Dicho porcentaje resulta más elevado al que fuera reportado en adolescentes de 12 a 14 años matriculados en una escuela básica rural cubana, donde la pérdida de primeros molares fue del $19,4 \%{ }^{(25)}$. En el estudio de Brennan et al. ${ }^{(11)}$ se ha reportado que aquellas personas con educación terciaria y nivel de ingresos familiares elevados, tenían menos dientes cariados y perdidos, con un índice CPO-D más bajo.

\section{CONCLUSIÓN}

En los adolescentes de la escuela de Pirayú, el nivel de conocimientos sobre salud oral no presentó relación con el índice de caries que tuvieron ni con el nivel de severidad de caries de riesgo bajo, pero un poco menos de la mitad perdió al menos un diente permanente. El nivel de conocimientos sobre salud oral fue suficiente en un poco más de la mitad. Se deben reforzar los programas de prevención primaria y secundaria, a fin de evitar pérdidas dentarias a temprana edad.

\section{REFERENCIAS BIBLIOGRÁFICAS}

1. Organización Mundial de la Salud. Desarrollo en la adolescencia [Internet]. 2018 [citado 21 de noviembre de 2018]. Recuperado a partir de: https://www.who.int/mate rnal_child_adolescent/topics/adolescence/dev/es/

2. Mafla AC. Adolescencia: cambios bio-psicosociales y 
salud oral. Colomb Med. 2008;39(1):41-57.

3. Agaku IT, Olutola BG, Adisa AO, Obadan EM, Vardavas CI. Association between unmet dental needs and school absenteeism because of illness or injury among U.S. school children and adolescents aged 6-17years, 2011-2012. Prev Med (Baltim). 2015;72:83-8.

4. Çolakoğlu N, Has E. The Evaluation of the Effects of Socio-Demographic Factors on Oral and Dental Health: A Study on the Ages 6.-12. Procedia - Soc Behav Sci. 2015;195:1278-87.

5. Oliveira DC, Ferreira FM, de Almeida Castro Morosini I, Torres-Pereira CC, Paiva SM, Fraiz FC. Impact of oral health status on the oral health-related quality of life of brazilian male incarcerated adolescents. Oral Health Prev Dent. 2015;13(5):417-25.

6. Cerón Bastidas XA. Relación de calidad de vida y salud oral en la poblacion adolescente. CES Odontol. 2018;31(1):38-46.

7. Pan American Health Organization. Health in the Americas+, 2017 Edition. Summary: Regional Outlook and Country Profiles. Whasington D.C.: PAHO;2017.248 p.

8. Sanabria-Castellanos CM, Suárez-Robles MA, EstradaMontoya JH. Relación entre determinantes socioeconómicos, cobertura en salud y caries dental en veinte países. Rev Gerenc y Políticas Salud. 2015;14(28):161-89.

9. Dirección de Salud Bucodental República del Paraguay. Ministerio de Salud Pública y Bienestar Social. Encuesta Nacional de salud bucodental del Paraguay 2017. MSPyBS; 2017.

10. Frazao P, Marques D. Effectiveness of a community health worker program on oral. Public Health. 2009;43(3):1-8.

11. Brennan D, Spencer J, Roberts-Thomson K. Dental knowledge and oral health among middle-aged adults. Aust N Z J Public Health. 2010;34(5):472-5.

12. Taniguchi-Tabata A, Ekuni D, Mizutani S, YamaneTakeuchi M, Kataoka K, Azuma T, et al. Associations between dental knowledge, source of dental knowledge and oral health behavior in Japanese university students: A cross-sectional study. PLoS One. 2017;12(6):1-11.

13. Torres Mahy L, Pérez Campos YE, Pascual Casamayor D, Noa Deyá Y. Modificación de conocimientos sobre salud bucal en adolescentes de la Secundarios. MEDISAN. 2015;19(3):365-73.
14. Ferreira e Ferreira E, Tomita NE, Dalben da Silva G. Accumulated knowledge and prevention practices in oral health. Braz Oral Res. 2014;28(1):1-8.

15. Dho M. Conocimientos de salud bucodental en relación con el nivel socioeconómico en adultos de la ciudad de Corrientes, Argentina. Rev Fac Nac Salud Pública. 2015;33(3):361-9.

16. Mehmood R, Rasheed S, Ijaz M. Knowledge , Attitude and Practice Regarding Oral Health Among Secondary School Students of Azad Kashmir, Pakistan. Pakistan J Public Heal. 2018;8(1):32-6.

17. Tascón JE, Alonso Cabrera G. Creencias sobre caries e higiene oral en adolescentes del. Valle del Cauca. Colomb Med. 2005;36(2):73-8.

18. Granville Garcia AF, Fernandes LV, Simões Farias TS, D'Ávila S, Menezes VA. Adolescents ' knowledge of oral health : a population-based study. Rev Odonto Ciência. 2010;25(4):361-6.

19. Rodríguez Vilchis LE, Contreras Bulnes R, Arjona Serrano J, Soto Mendieta R, Alanís Tavira J. Prevalencia de caries y conocimientos sobre salud-enfermedad bucal de niños (3 a 12 años) en el Estado de México. Rev ADM. 2006;63(5):170-5.

20. Veiga N, Pereira C, Amaral O. Prevalence and Determinants of Dental Caries in Portuguese Children. Procedia - Soc Behav Sci. 2015;171:995-1002.

21. Borges TS, Schwanke NL, Reuter CP, Neto LK, Burgos MS. Fatores associados à cárie: pesquisa de estudantes do sul do Brasil. Rev Paul Pediatr. 2016;34(4):489-94.

22. Žemaitiene M, Grigalauskiene R, Vasiliauskiene I, Saldunaite K, Razmiene J, Slabšinskiene E. Prevalence and severity of dental caries among 18-year-old Lithuanian adolescents. Med. 2016;52(1):54-60.

23. Hans R, Thomas S, Dagli R, Bhateja GA, Sharma A, Singh A. Oral health knowledge, Attitude and practices of children and adolescents of orphanages in jodhpur city rajasthan, India. J Clin Diagnostic Res. 2014;8(10):ZC22-ZC25.

24. Dopico M, Castro C. Importancia del primer molar permanente y consecuencias clínicas de su pérdida en edades tempranas del desarrollo. Acta odontológica Venez. 2015;XLV(2):23-37.

25. Sánchez D, Pons Y, Betancourt A, Santateresa A. Pérdida del primer molar permanente : factores de riesgo y salud bucodental en adolescentes. Rev Finlay. 2017;7(1):17-25. 